\title{
Nested Case-Control Study of Corin Combined with sFlt-I/PLGF in Predicting the Risk of Preeclampsia
}

\author{
Mei Liu ${ }^{1,2}$ \\ Rui-Bo Wang' \\ Jian-Hong Xing ${ }^{2}$ \\ Ying-Xue Tang ${ }^{3}$ \\ 'Chinese Medicine College, Shandong \\ University of Traditional Chinese \\ Medicine, Jinan, 250355, Shandong, \\ People's Republic of China; ${ }^{2}$ Department \\ of Obstetrics, Affiliated Hospital of \\ Shandong University of Traditional \\ Chinese Medicine, Jinan, 2500II, \\ Shandong, People's Republic of China; \\ ${ }^{3}$ Postgraduate Department, Shandong \\ University of Traditional Chinese \\ Medicine, Jinan, 250355, Shandong
}

Background: Preeclampsia (PE), a serious pregnancy disorder, is responsible for maternal and fetal mortality worldwide. At present, numerous candidate biomarkers have been studied to predict PE.

Objective: To explore the role of Corin in PE risk prediction and then evaluate the predictive ability of soluble vascular endothelial growth factor receptor-1 (sFlt-1), placenta growth factor (PLGF), and sFlt-1/PLGF after the addition of Corin.

Methods: A total of 135 pregnant women from Affiliated Hospital of Shandong University of Traditional Chinese Medicine participated in this study in their first trimester. A nested case-control study was conducted and all subjects were divided into PE groups $(n=46)$ and controls $(n=89)$. The levels of PLGF, sFlt-1, sFlt-1/PLGF ratio, and Corin of the two groups at 12-16 weeks of gestation were measured and analyzed. Receiver operating characteristic (ROC) curve, net reclassification index (NRI) and integrated discrimination index (IDI) were calculated to evaluate the predictive ability of various biomarkers.

Results: The concentrations of sFlt-1, sFlt-1/PLGF, and Corin in PE group were significantly higher than that in controls, while the concentration of PLGF in the PE group was lower. The area under curve (AUC) of sFlt-1, PLGF and sFlt-1/PLGF for predicting PE was $0.786,0.719$ and 0.866 , respectively. Combined with Corin, the prediction ability of the above biomarkers could be improved to $0.876,0.847$, and 0.897 , respectively. Corin in combination with sFlt-1/PLGF resulted in improvements with $12.6 \%$ being reclassified and a resulting NRI of $0.142(0.020 \sim 0.263)$ and IDI of $0.087(0.037 \sim 0.137)$.

Conclusion: The addition of Corin to sFlt-1, PLGF and sFlt-1/PLGF can improve the ability of each marker to predict PE risk. Corin in combination with sFlt-1/PLGF can be used as ideal markers to identify the pregnant women who subsequently develop PE, which will help in risk stratification and better therapeutic management.

Keywords: preeclampsia, Corin, soluble vascular endothelial growth factor receptor-1, placental growth factor, prediction

\section{Introduction}

Preeclampsia $(\mathrm{PE})$ is a pregnancy-specific disorder that occurred in pregnant women after 20-week gestation with symptoms of hypertension, proteinuria, and edema. This disease has the characteristics of insidious onset, rapid progression, and poor prognosis and the incidence is as high as $5 \% \sim 10 \%$, which remains a leading cause of maternal mortality and is a major threat to maternal and fetal health. $^{1-4}$ Numerous studies demonstrating that early detection of high-risk
Porrespondence: Ying-Xue Tang University of Traditional Chinese Medicine, Changqing Campus of Shandong University of Traditional Chinese Medicine, Jinan, 250355, Shandong, People's Republic of China Tel +8653 I89628067

Email doctortyx@163.com 
populations is of great significance for preventing and treating PE and improving pregnancy outcomes. ${ }^{2,5}$ Therefore, it may be essential to find new predictive biomarkers of PE. Even though the exact etiology and pathophysiology remain elusive, further research suggests that the dysfunction of placental trophoblast and the damage of maternal vascular endothelium are the main pathogenesis. ${ }^{6,7}$ The biomarkers in relation to placental and vascular injury, including soluble vascular endothelial growth factor receptor-1 (sFlt-1) and placenta growth factor (PLGF), have shown better precision and practical values, which has greatly advanced the prediction and early diagnosis of PE. A series of studies suggest that the comprehensive evaluation model of sFlt-1 combined with PLGF is more sensitive than alone and has good prospective application in clinical work, but further research is still needed to improve the feasibility and effectiveness of prediction. $^{8-10}$

Recent developments in the field of the pathogenesis of PE have led to the discovery of more biomarkers. To date, there have been no studies with specificity and sensitivity required for clinical applications, and the difference between different studies is obvious. ${ }^{11}$ More recent studies have demonstrated that cardiac serine protease-Corin plays a crucial role in development of disease. Indeed, silencing Corin in mice will generate the cardinal manifestations of human preeclampsia as hypertension and proteinuria, suggesting the level of Corin in the blood of the mother may be a predictive marker for PE. ${ }^{12-14}$

Yet the relationship between Corin and PE still needs attention and only a few studies have investigated the predictive utility of sFlt-1 and PLGF the introduction of Corin. The purpose of this study is to identify women at high risk of developing PE by using appropriate prediction models.

\section{Methods}

\section{Patients}

A total of 956 pregnant women with singleton pregnancy attended the obstetrics department of Affiliated Hospital of Shandong University of Traditional Chinese Medicine from April 2016 to April 2019 in their first trimester. The gestational age was calculated from the last menstrual period and confirmed by ultrasound ultrasonography between 10 and 12 weeks of gestation. Exclusion criteria: Women suffer from diabetes, morbid obesity (body mass index $>40 \mathrm{~kg} / \mathrm{m}^{2}$ ), hypertension, cardiovascular system diseases, systemic lupus erythematosus, metabolic diseases, liver and kidney diseases. A nested case-control study was performed and all subjects were divided into PE group $(n=46)$ and normal controls $(n=89)$ according to pregnancy outcome. The controls consisted of singleton pregnancy women (matched on age) without hypertension, diabetes, hyperthyroidism, and other pre-pregnancy complications. This study was approved by the ethical committee of Affiliated Hospital of Shandong University of Traditional Chinese Medicine and conducted in accordance with the Declaration of Helsinki. All subjects signed informed consent before the investigation.

\section{Biomarkers Detection}

Baseline data were collected, including age, systolic blood pressure, diastolic blood pressure, body mass index, gestational age, and smoking history. A total of $4 \mathrm{~mL}$ fasting venous blood was obtained from all 135 participants in the morning at 12-16 weeks of gestation. After centrifugation for $15 \mathrm{~min}$ at $1500 \times \mathrm{g}$, the serum was separated and then immediately stored in $-80^{\circ} \mathrm{C}$. The serum concentrations of Corin, PLGF, and sFlt-1 were measured by ELISA analysis, and the kit was purchased from Boster Biological Technology co.ltd.

\section{Clinical Definitions}

According to the criteria of the American College of Obstetricians and Gynecologists (ACOG), PE was defined when blood pressure $\geq 140 / 90 \mathrm{~mm} \mathrm{Hg}$ that developed after 20 weeks' gestation in a previously normotensive woman, with proteinuria (24-hour urinary protein $\geq 300 \mathrm{mg}$ ). Additionally, severe preeclampsia was diagnosed with any of the following findings: systolic blood pressure $\geq 160 \mathrm{mmHg}$, or diastolic blood pressure $\geq 110$ $\mathrm{mmHg}$; platelet count $<100,000 / \mu \mathrm{L}$; elevated liver enzymes; severe persistent right upper quadrant or epigastric pain; renal insufficiency; pulmonary edema; new-onset cerebral or visual disturbances. Fetal growth restriction (FGR) is defined as the 10th percentile with an estimated body weight less than gestational age.

\section{Statistical Analysis}

All analyses were performed using SPSS 22.0, MedCalc version 10.2.0.0, and $\mathrm{R}$ package version 3.6.2. Normaldistributed data were expressed as $\bar{x} \pm s$ and compared using the Student's $t$-test. Non-normal data were described by median and quartiles. Comparison was conducted in two groups with the Mann-Whitney $U$-test. The $\chi^{2}$ test 
was used for the comparison of categorical variables. Receiver operating characteristic (ROC) curve, net reclassification index (NRI) and integrated discrimination index (IDI) were calculated to evaluate the predictive ability of Corin, PLGF, sFlt-1, alone and in combination for PE. Statistical significance was regarded as $P<0.05$.

\section{Results}

\section{Comparison of Clinical Characteristics Between the Two Groups}

Of the 956 pregnant women, 46 subjects subsequently developed PE with the incidence of $4.8 \%$, of which 19 subjects (19/46) were severe, 27 subjects (27/46) were mild, and 23 subjects (23/46) complicated by FGR. There were 36 primiparas and 10 multiparas in the PE group, and 60 primiparas, 29 multiparas in the normal control group. The age range of the subjects was from 23 to 38 years with gestational age from 26 to 41 weeks. Diagrammatic flow of study design were listed in the Figure 1. The clinical characteristics of patients in the PE group and normal control group were listed in Table 1. Women developed PE showed a significant increase in body mass index, systolic blood pressure, diastolic blood pressure, and incidence of Caesarean delivery, but a decrease in gestational age as compared with controls $(P<0.05)$. There was no significant difference in age and rates of smoking between the two groups $(P>0.05)$.

\section{Comparison of Biomarker Levels Between the Two Groups}

The results showed that the concentrations of sFlt-1 [3366.1 (2730.7, 4553.0) vs 2561.8 (2057.4, 3034.5)], sFlt-1/PLGF $[19.5(13.9,26.5)$ vs $10.6(8.2,14.1)]$ and Corin $[1184.4(932.6,2966.9)$ vs $893.0(719.0,1106.9)]$ in the PE group were significantly higher than that in the controls, while the PLGF concentration [203.1 (155.6, 248.9) vs $166.0(132.1,197.6)]$ was significantly lower $(\mathrm{P}<0.001)$. The biomarker expression levels of $\mathrm{PE}$ group and controls were presented in Figure 2.

\section{Predictive Value of Biomarkers for PE}

As shown in Figure 3A, the Area under curve (AUC) of sFlt1/PLGF was $0.866(0.796 \sim 0.918)$, obviously higher than that of sFlt-1[0.786 $(0.707 \sim 0.852)]$, PLGF $[0.719(0.635 \sim$ 0.793)] and Corin $[0.761(0.680 \sim 0.830)]$, respectively. After combined with Corin, the AUC of sFlt-1, PLGF, and sFlt-1/PLGF can be increased to $0.876(0.808 \sim 0.926)$, $0.847(0.775 \sim 0.903)$, and $0.897(0.833 \sim 0.943)$, respectively (Figure 3B). Compared with sFlt-1/PLGF, the AUC of sFlt-1/PLGF in combination with Corin increased by 0.031 , but the difference was not statistically significant $(P=0.141)$.

\section{The Predictive Ability of Biomarkers with the Introduction of Corin}

According to the Youden index, the risk of PE was divided into low, medium, and high: $<40 \%, 40 \% \sim 60 \%$, and

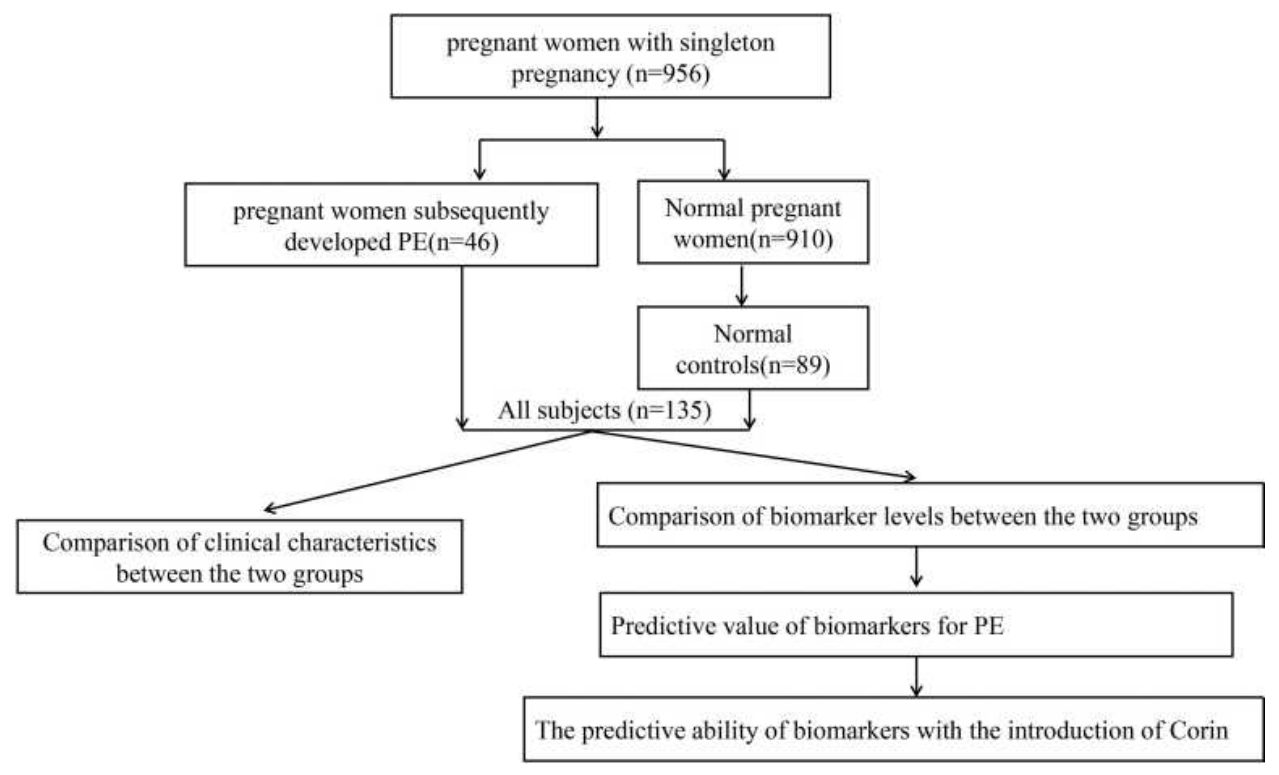

Figure I Diagrammatic flow of study design. 
Table I Clinical Characteristics of PE and Controls

\begin{tabular}{|c|c|c|c|}
\hline & PE $(n=46)$ & Controls $(n=89)$ & $\boldsymbol{P}$ \\
\hline Age, year & $30.0(26.0,33.0)$ & $29.0(26.0,32.0)$ & 0.111 \\
\hline Body mass index, $\mathrm{kg} / \mathrm{m}$ & $23.4(22.2,24.7)$ & $21.4(20.1,22.6)$ & $<0.001$ \\
\hline Systolic blood pressure, $\mathrm{mmHg}$ & $152.5(139.5,158.5)$ & $113(108,121)$ & $<0.001$ \\
\hline Diastolic blood pressure, $\mathrm{mmHg}$ & $97.5(90,108.5)$ & $75(70,81)$ & $<0.001$ \\
\hline Current smoker, $\%$ & I $(2.17 \%)$ & $2(2.22 \%)$ & 0.981 \\
\hline Gestational age at delivery, weeks & $37.1(36.2,38.2)$ & $39.2(38.0,40.3)$ & $<0.001$ \\
\hline Caesarean Delivery, \% & $35(76.1 \%)$ & $23(25.8 \%)$ & $<0.001$ \\
\hline Primiparas, \% & $36(78.26 \%)$ & $60(67.41 \%)$ & 0.188 \\
\hline
\end{tabular}

Notes: Data is presented as medians and quartiles or as percentage $(n)$.

$\geq 60 \%$. The result showed that the Corin in combination with sFlt-1 improved only the IDI while Corin in combination with PLGF or sFlt-1/PLGF provided the significant increases in both the NRI and IDI. When Corin combined with PLGF, 39.3\% of the individuals were reclassified and the resulting NRI and IDI were 0.328 and 0.199
A

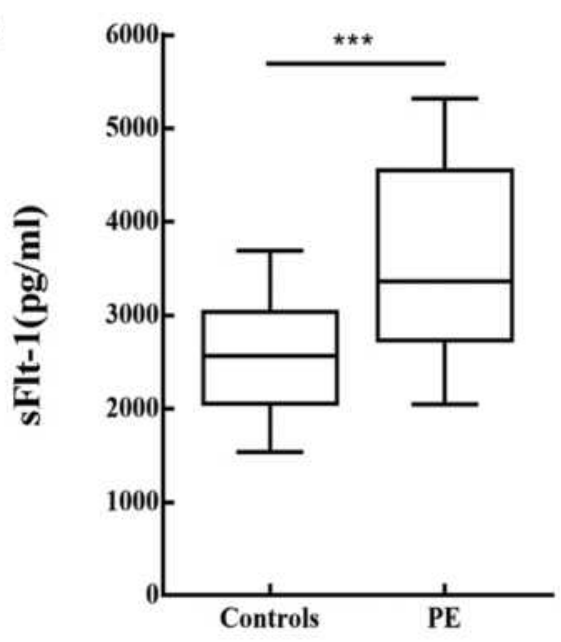

C

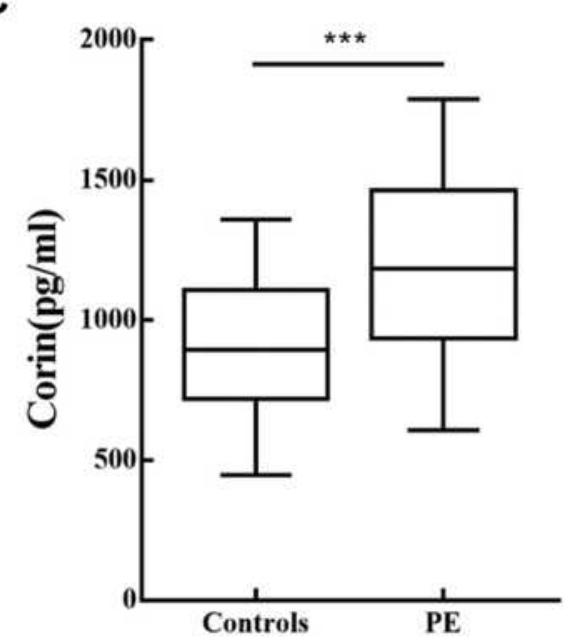

B

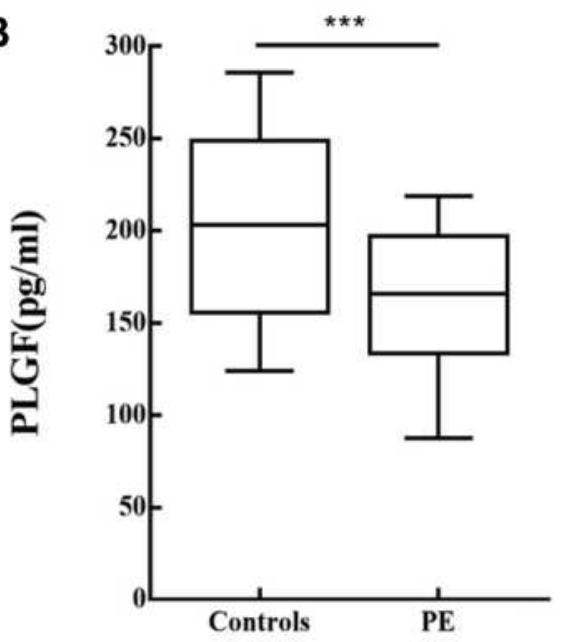

D

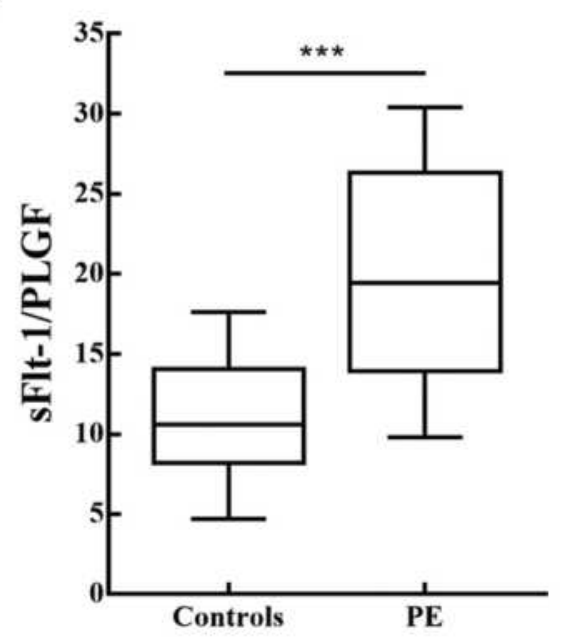

Figure 2 Biomarker levels in PE and Controls at I4-16 weeks of gestation. (A) sFlt-I; (B) PLGF; (C) sFIt-I/PLGF; (D) Corin; Compared with the controls: ***P<0.00I. 
A

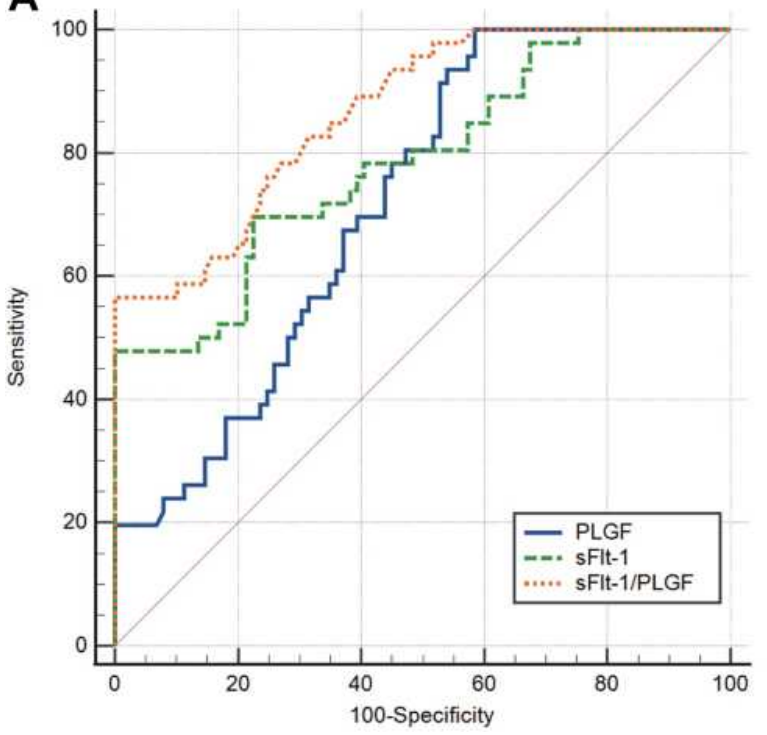

B

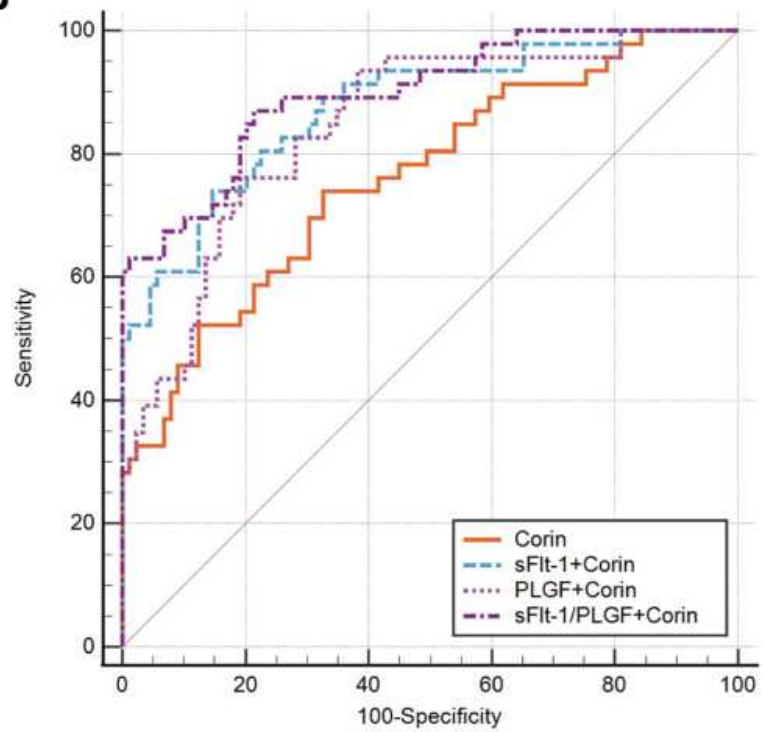

Figure 3 ROC curve of sFlt-I, PLGF, sFlt-I/PLGF and Corin, alone and in combination for prediction of PE (A) sFlt-I, PLGF and sFIt-I/PLGF alone; (B) Combined with Corin.

respectively. Adding Corin to the sFlt-1/PLGF resulted in improvements with $12.6 \%$ being reclassified and a resulting NRI of 0.142 and IDI of 0.087 (Table 2).

\section{Discussion}

Preeclampsia is a serious and potentially life-threatening disease in pregnancy. Statistics show that PE patients have suffered damage at varying degrees before the onset of their clinical manifestations. ${ }^{15}$ Therefore, it is important to establish an early prediction model and screen high-risk pregnant women for disease prevention and treatment. There are many prediction methods for PE, including manifestations and biomarkers, but these sensitivity and specificity are low, thus limiting the clinical application. In-vitro and in-vivo studies have elucidated that abnormal expression of Corin and gene mutations can lead to invasive abnormity of the trophoblast and impairment of uterine spiral artery that can result in PE. ${ }^{16,17}$ This study retrospectively analyzed the alterations of biomarkers in PE and normal pregnant women and then explore the role of Corin combined with traditional biomarkers in the pathogenesis of PE.

Biomarkers detection is one of the important methods for predicting PE. It can determine a credible early disease assessment during the asymptomatic stage of pregnant women and effectively monitor high-risk groups. Since PE is associated with endothelial cell dysfunction and placental abnormalities, studies on relevant biomarkers are imperative for the early prediction of PE. ${ }^{18-20}$ The correlation between alterations in
PLGF and sFlt-1 concentrations with preeclampsia disease activity was confirmed in several studies. Zhao et $\mathrm{al}^{21}$ reported that serum sFlt-1 is more pronounced in pregnant women with PE, which binds and neutralizes PLGF, and lead to a decrease in bioavailable PLGF. This contributes to increase vascular permeability and accelerates endothelial and vascular dysfunction. Similarly, the results of this study showed that compared with the controls, the serum sFlt-1 concentrations in PE pregnant women were increased, whereas the PLGF concentrations were decreased, suggesting that sFlt-1 and PLGF may be involved in the generation and development of PE. ROC curve analysis showed that the AUC for sFlt-1 and PLGF was more than 0.7 , which was helpful in the prediction and diagnosis of PE, but the prediction effect was not obvious.

Moreover, meta-analysis indicated that the sensitivity and specificity of individual biomarkers alone are moderate, while a composite measure incorporating sFlt-1 and PLGF is more predictive of $\mathrm{PE}$ than either measure alone. $^{22}$ According to current data, the sFlt-1/PLGF ratio gives a much-improved sensitivity of $89 \% \sim 100 \%$ and the specificity of $81 \% \sim 98 \% .^{23}$ In the present study, the ROC curve also showed that the AUC of sFlt-1/PLGF was higher than either of them and the results were consistent with the above research. As sFlt-1/PLGF shows significant alteration in the third trimester, it is necessary to combine relevant indicators in order to improve the accuracy of prediction in the first trimester and the second trimester. Therefore, a new serological index-Corin was analyzed 
Table 2 Reclassification Comparison of Each Biomarker Combined with Corin

\begin{tabular}{|c|c|c|c|c|}
\hline \multirow[t]{2}{*}{ Biomakers } & \multicolumn{4}{|c|}{ Combined with Corin } \\
\hline & $<40 \%$ & $40 \% \sim 60 \%$ & $\geq 60 \%$ & \% Reclassified \\
\hline \multicolumn{5}{|l|}{ sFlt-I } \\
\hline$<40 \%$ & 74 & 11 & 5 & 18 \\
\hline $40 \% \sim 60 \%$ & 10 & 6 & 7 & 74 \\
\hline$\geq 60 \%$ & 0 & 2 & 20 & 9 \\
\hline NRI & \multicolumn{4}{|c|}{$0.140(-0.042 \sim 0.322), P=0.131$} \\
\hline IDI & \multicolumn{4}{|c|}{$0.146(0.076 \sim 0.215), P<0.001$} \\
\hline \multicolumn{5}{|l|}{ PLGF } \\
\hline$<40 \%$ & 68 & 9 & 8 & 20 \\
\hline $40 \% \sim 60 \%$ & 18 & 8 & 14 & 80 \\
\hline$\geq 60 \%$ & 0 & 4 & 6 & 40 \\
\hline NRI & \multicolumn{4}{|c|}{$0.328(0.093 \sim 0.562), P=0.006$} \\
\hline IDI & \multicolumn{4}{|c|}{$0.199(0.122 \sim 0.277), P<0.001$} \\
\hline \multicolumn{5}{|l|}{ sFIt-I/PLGF } \\
\hline$<40 \%$ & 83 & 6 & I & 8 \\
\hline $40 \% \sim 60 \%$ & 6 & 9 & 4 & 53 \\
\hline$\geq 60 \%$ & 0 & 0 & 26 & 0 \\
\hline NRI & \multicolumn{4}{|c|}{$0.142(0.020 \sim 0.263), P=0.022$} \\
\hline IDI & \multicolumn{4}{|c|}{$0.087(0.037 \sim 0.137), \mathrm{P}<0.00 \mathrm{I}$} \\
\hline
\end{tabular}

in our study. Several studies evaluated the relationship between Corin and hypertensive disorder during pregnancy and could be applied for the prediction of PE. ${ }^{24,25}$ The serum Corin concentrations increase slightly in normal healthy pregnant women, while dramatically alter in women with PE as early as 12 weeks of gestation, indicating that Corin may have a predictive role in the pathogenic process of PE. Rong et $\mathrm{al}^{26}$ have reported that the women who subsequently develop PE have a significant rise in the Corin concentrations in early pregnancy and the similar alterations in Corin was observed in this study. Relative to controls, women in the PE group exhibited higher Corin with AUC of 0.761, which provided evidence for the clinical application of serum Corin in predicting PE. In order to evaluate the impact of Corin on the utility of sFlt1, PLGF, and sFlt-1/PLGF, Corin in combination with other biomarkers was analyzed and results showed the increased predictive ability of combination with Corin.
Although Corin in combination with sFlt-1/PLGF showed the highest AUC of 0.897 , there was no significant difference compared with sFlt-1/PLGF. Our data indicated that the impact of the addition of Corin on AUC was not significant, but the number of individuals reclassified and NRI caused by the new predictive model needs to be further determined. Conceptually, NRI is a statistical method to evaluate the predictive ability of the new and traditional models, and IDI is used to reflect the overall improvement of the new model. After the introduction of Corin, the NRI and IDI of sFlt-1/PLGF were significantly increased, which indicated that the prediction ability of sFlt-1/PLGF was improved with the addition of Corin, and the difference was statistically significant. The results of this study identified sFlt-1/PLGF in combination with Corin during the early second trimester as a useful tool in the clinical prediction, and the combination gave high predictive values in women at risk of developing PE.

The main limitations of our study include: (1) Only 135 participants were enrolled and there may be a selection bias; (2) There are fewer types of serological markers analyzed, and others were not included in the test due to methodological limitations; (3) The pregnant women at a specific time were included, and women during the whole pregnancy need to be confirmed by subsequent studies. (4) The model is not validated in a different cohort.

\section{Conclusion}

In summary, Corin can slightly improve the prediction ability of sFlt-1 and PLGF for PE. Corin in combination with sFlt-1/PLGF may be used as ideal biomarkers for subsequent PE in pregnant women develop, contributing to risk stratification and better treatment management.

\section{Abbreviations}

PE, Preeclampsia; sFlt-1, soluble vascular endothelial growth factor receptor-1; PLGF, placenta growth factor; ROC, receiver operating characteristic; NRI, net reclassification index; IDI, integrated discrimination index; FGR, fetal growth restriction; AUC, area under curve; ANP, atrial natriuretic peptide.

\section{Ethics Approval and Consent to Participate}

This work was supported by the ethics committee of Affiliated Hospital of Shandong University of Traditional Chinese Medicine. 


\section{Acknowledgment}

The authors would like to thank the staff and participants for their contribution to this study.

\section{Author Contributions}

All authors made substantial contributions to conception and design, acquisition of data, or analysis and interpretation of data; took part in drafting the article or revising it critically for important intellectual content; agreed to submit to the current journal; gave final approval of the version to be published; and agree to be accountable for all aspects of the work.

\section{Funding}

The study was funded by the Medical Health Science and Technology Project of Shandong Province (2017WS733), TCM Science and Technology Project of Shandong Province(2019-0118) and Science and Technology Project of Shandong Provincial Health Care Technology Association(SDBJKT20170004). Shandong University of traditional Chinese medicine research and innovation team project (2018-10 traditional Chinese Medicine theory application and efficacy screening team).

\section{Disclosure}

The authors report no conflicts of interest in this work.

\section{References}

1. Steegers EAP, von Dadelszen P, Duvekot JJ, Pijnenborg R. Preeclampsia. Am J Pathol. 2010;176(2):710-720. doi:10.2353/ ajpath.2010.090513

2. Ramos JGL, Sass N, Costa SHM. Preeclampsia. Rev Bras Ginecol Obstet. 2017;39(9):496-512. doi:10.1055/s-0037-1604471

3. Filipek A, Jurewicz E. [Preeclampsia - a disease of pregnant women]. Postepy Biochem. 2018;64(4):232. English. doi:10.18388/ pb.2018_146

4. Powel JE, Rosenthal E, Roman A, Chasen ST, Berghella V. Preeclampsia and low sodium (PALS): a case and systematic review. Eur J Obstet Gynecol Reprod Biol. 2020;249:14-20. doi:10.1016/j. ejogrb.2020.03.052

5. Sibai BM, Stella CL. Diagnosis and management of atypical preeclampsia-eclampsia. Am J Obstet Gynecol. 2009;200(5):481.e17. doi:10.1016/j.ajog.2008.07.048

6. Rana S, Lemoine E, Granger JP, Ananth Karumanchi S. Preeclampsia: pathophysiology, challenges, and perspectives. Circ Res. 2019;124 (7):1094-1112. doi:10.1161/CIRCRESAHA.118.313276

7. El-Sayed AAF. Preeclampsia: a review of the pathogenesis and possible management strategies based on its pathophysiological derangements. Taiwan J Obstet Gynecol. 2017;56(5):593-598. doi:10.1016/j.tjog.2017.08.004

8. Herraiz I, Llurba E, Verlohren S, Galindo A; Spanish Group for the Study of Angiogenic Markers in Preeclampsia. Update on the diagnosis and prognosis of preeclampsia with the aid of the sFlt-1/PIGF ratio in singleton pregnancies. Fetal Diagn Ther. 2018;43(2):81-89. doi:10.1159/000477903
9. Lecarpentier É, Vieillefosse S, Haddad B, et al. Placental Growth Factor (PlGF) and sFlt-1 during pregnancy: physiology, assay and interest in preeclampsia. Ann Biol Clin. 2016;74(3):259-267.

10. Cui L, Shu C, Liu Z, et al. The expression of serum sEGFR, sFlt-1, sEndoglin and PLGF in preeclampsia. Pregnancy Hypertens. 2018;13:127-132. doi:10.1016/j.preghy.2018.05.011

11. Lecarpentier E, Tsatsaris V. Angiogenic balance (sFlt-1/PlGF) and preeclampsia. Ann Endocrinol. 2016;77(2):97-100. doi:10.1016/j. ando.2016.04.007

12. Zhou Y, Wu Q. Role of corin and atrial natriuretic peptide in preeclampsia. Placenta. 2013;34(2):89-94. doi:10.1016/j. placenta.2012.11.016

13. Chalova KI, Pehlivanov BK, Amaliev IG, Amaliev GI, Raycheva RD, Ivanovska MV. Maternal serum concentrations of corin, endoglin, PP13, and sFlt-1 and their changes with advancement of pregnancy and correlation with doppler of uterine arteries. Folia Med. 2018;60(4):558-564. doi:10.2478/folmed-2018-0025

14. Cui Y, Wang W, Dong N, et al. Role of corin in trophoblast invasion and uterine spiral artery remodelling in pregnancy. Nature. 2012;484 (7393):246-250. doi:10.1038/nature10897

15. Méhats C, Miralles F, Vaiman D. [New perspectives on preeclampsia]. Med Sci. 2017;33(12):1079-1088. English.

16. Hui L, Zhang Y, Qingyu W. Role of corin in the regulation of blood pressure. Curr Opin Nephrol Hypertens. 2017;26(2):67-73. doi:10.1097/MNH.0000000000000297

17. Miyazaki J, Nishizawa H, Kambayashi A, et al. Increased levels of soluble corin in pre-eclampsia and fetal growth restriction. Placenta. 2016;48:20-25. doi:10.1016/j.placenta.2016.10.002

18. Verlohren S, Perschel FH, Thilaganathan B, et al. Angiogenic markers and cardiovascular indices in the prediction of hypertensive disorders of pregnancy. Hypertension. 2017;69(6):1192-1197. doi:10.1161/HYPERTENSIONAHA.117.09256

19. Taravati A, Tohidi F. Comprehensive analysis of oxidative stress markers and antioxidants status in preeclampsia. Taiwan J Obstet Gynecol. 2018;57(6):779-790. doi:10.1016/j.tjog.2018.10.002

20. Sonek J, Krantz D, Carmichael J, et al. First-trimester screening for early and late preeclampsia using maternal characteristics, biomarkers, and estimated placental. Am J Obstet Gynecol. 2018;218(1):126. e1-126.e13. doi:10.1016/j.ajog.2017.10.024

21. Zhao M, Zhu Z, Liu C, Zhang Z. Dual-cutoff of sFlt-1/PlGF ratio in the stratification of preeclampsia: a systematic review and meta-analysis. Arch Gynecol Obstet. 2017;295(5):1079-1087. doi:10.1007/s00404-017-4302-3

22. Doherty A, Carvalho JCA, Drewlo S, et al. Altered hemodynamics and hyperuricemia accompany an elevated sFlt-1/PlGF ratio before the onset of early severe preeclampsia. J Obstet Gynaecol Can. 2014;36(8):692-700. doi:10.1016/S1701-2163(15)30511-9

23. Liu Y, Zhao Y, Ailing Y, Zhao B, Gao Y, Niu H. Diagnostic accuracy of the soluble fms-like tyrosine kinase-1/placental growth factor ratio for preeclampsia: a meta-analysis based on 20 studies. Arch Gynecol Obstet. 2015;292(3):507-518. doi:10.1007/s00404-015-3671-8

24. Khalil A, Maiz N, Garcia-Mandujano R, Elkhouli M, Nicolaides KH. Longitudinal changes in maternal corin and mid-regional proatrial natriuretic peptide in women at risk of pre-eclampsia. Ultrasound Obstet Gynecol. 2015;45(2):190-198. doi:10.1002/uog.14685

25. Armaly Z, Assady S, Abassi Z. Corin: a new player in the regulation of salt-water balance and blood pressure. Curr Opin Nephrol Hypertens. 2013;22(6):713-722. doi:10.1097/01. mnh.0000435609.35789.32

26. Rong Y, Han X, Zhang X, Wang Y, Wang T. Circulating soluble corin as a potential biomarker for cardiovascular diseases: a translational review. Clin Chim Acta. 2018;485:106-112. doi:10.1016/j. cca.2018.06.036 


\section{Publish your work in this journal}

The International Journal of General Medicine is an international, peer-reviewed open-access journal that focuses on general and internal medicine, pathogenesis, epidemiology, diagnosis, monitoring and treatment protocols. The journal is characterized by the rapid reporting of reviews, original research and clinical studies across all disease areas. The manuscript management system is completely online and includes a very quick and fair peer-review system, which is all easy to use. Visit http://www.dovepress.com/ testimonials.php to read real quotes from published authors. 